
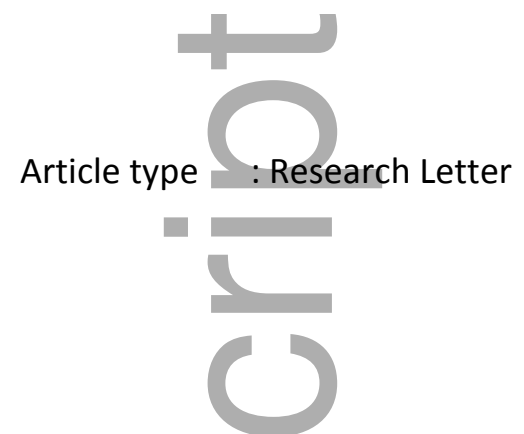

\title{
A new clinical variant of acquired reactive perforating dermatosis-like bullous pemphigoid
}

Franziska Schauer $^{1}$, Johannes Steffen Kern ${ }^{1,2}$, Oana Virtic ${ }^{1}$, Kristin Technau-Hafsi ${ }^{1}$, Frank Meiss ${ }^{1}$, Kaethe Thoma ${ }^{1}$, Ioannis Athanasiou ${ }^{1}$, Cassian Sitaru', Giovanni Di Zenzo ${ }^{3}$, Kentaro Izumi $^{4}$, Wataru Nishie ${ }^{4}$, Hiroshi Shimizu ${ }^{4}$, Leena Bruckner-Tuderman ${ }^{1}$, Dimitra Kiritsi ${ }^{1}$

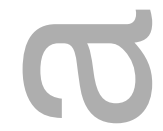

${ }^{1}$ Department of Dermatology, Medical Center- University of Freiburg, Faculty of Medicine, University of Freiburg, Freiburg, Germany

${ }^{2}$ Royal Melbourne Hospital, Parkville and Box Hill Hospital - Monash University Eastern Health Clinical School, Box Hill, Victoria, Australia

${ }^{3}$ Laboratory of Molecular and Cell Biology, Istituto Dermopatico dell'Immacolata (IDI)-IRCCS, FLMM, Rome, Italy

${ }^{4}$ Department of Dermatology, Graduate School of Medicine, Hokkaido University, Sapporo, Japan

\section{The authors have no conflict of interest to disclose.}

Corresponding author: Dimitra Kiritsi

Email: dimitra.kiritsi@uniklinik-freiburg.de

This is the author manuscript accepted for publication and has undergone full peer review but has not been through the copyediting, typesetting, pagination and proofreading process, which may lead to differences between this version and the Version of Record. Please cite this article as doi: $10.1111 /$ bjd.17146

This article is protected by copyright. All rights reserved 


\section{ORCID:}

Dr Franziska Schauer (Orcid ID 0000-0001-5147-0123)

Dr Johannes S Kern (Orcid ID 0000-0001-6372-8048)

Dr Dimitra Kiritsi (Orcid ID : 0000-0002-2331-8981)

Dr Cassian Sitaru (ORCID ID: 0000-0002-0370-1270)

\section{Running head:}

Acquired reactive perforating dermatosis-like bullous pemphigoid

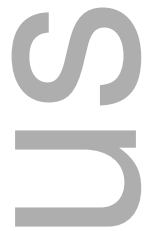

\section{Dear Editor}

Bullous pemphigoid (BP) is a blistering disorder associated with circulating autoantibodies against BP180/collagen XVII. As part of a prodromal stage patients might present with eczematous lesions or erythematous papules and prurigo- or urticaria-like erythema without any blistering ${ }^{1,2}$. Recent studies described four patients with clinical features resembling acquired reactive perforating dermatosis (ARPD) co-existing with $\mathrm{BP}^{3,4}$. They initially presented with papules and nodules with a central keratotic plaque; diabetes mellitus (DM) and hemodialysis thought of being causative. They developed only at a later time point blisters, and BP180 autoantibodies were found to bind at the DEJZ.

We here describe 5 previously unreported patients, which clinically and histologically resembled ARPD with simultaneous BP diagnosis. All patients were over 70 years of age with several concomitant disorders; and all except patient 1 were female. They all suffered from hypertension; patients 3 and 4 also had atrial fibrillation, whereas patients 1 and 3 also had type II DM with signs of diabetic nephropathy. Patient 2 had a history of breast cancer in remission, 11 years prior. We found elevated $\lg E$ levels and an elevated blood eosinophil count.

Patients 1-4 presented with a history of sudden severe itch and generalized papules and nodules with a central keratotic plaque without any blisters (Figure 1B). Patient 5 had at presentation the characteristic BP with large urticarial plaques and blisters covering large parts of her integument, which had developed 2.5 months ago (Figure 1B). After diagnosing $\mathrm{BP}$, she received topical treatment with clobetasolpropionate ointment, systemic prednisolone and mycophenolate mofetil. She showed a quick treatment response with reduced itch and healing of lesions. Six weeks later she presented again, now with the ARPD This article is protected by copyright. All rights reserved 
picture of papular lesions with central keratotic plugs on her legs, back and upper arms (Figure 1B).

In the biopsies taken from the papules and nodules in patients 1 to 4 , the histopathology showed a cup-shaped invagination of the epidermis filled with necrotic debris and vertically penetrating collagen bundles between dermis and epidermis with a dermal neutrophilic infiltrate (Figure 1C), which represents the histopathological hallmark of reactive perforating collagenosis $(\mathrm{RPC})^{5}$. Solely in the skin biopsy of patient 5 , with blistering at initial presentation, a subepidermal skin cleavage with dense eosinophilic infiltrate and dermal edema was found. She had the ARPD picture only at follow up. DIF was performed in all cases at first presentation. It showed the typical linear deposition of $\lg G$ and $\mathrm{C} 3 \mathrm{c}$ at the DEJZ. The indirect immunofluorescence showed IgG depositions on the blister roof, also in all patients (Supplemental Figure 1a). The presence of autoantibodies against the full-length form of BP180 has recently been linked to a non-inflammatory BP phenotype, accompanied by scant lesional eosinophilic infiltration ${ }^{6}$. ELISA diagnostics recognizing the NC16A domain did not detect BP180 IgG autoantibodies in patients 1, 3 and 4, but patients 1 and 3 had autoantibodies against the full-length BP180 (Figure 1B). Patients 2 and 4 also had BP230 antibodies. IgE autoantibodies are associated with increased BP severity. We analysed the sera for IgE autoantibodies to BP180 and BP230 and found low-level BP230 lgEautoantibodies in cases 2 and 4, while case 5 had low level BP180 and BP230 lgEautoantibodies. To investigate the pathogenic potential of autoantibodies in these patients, we have used a previously described ex vivo assay ${ }^{7}$. In contrast to serum from a reference BP patient, serum samples from patients evaluated in the present study showed different pathogenic potential in this assay, ranging from no split induction to different degrees of modest dermal-epidermal separation (Supplemental Figure 1b). While the somehow lower reactivity does certainly not fully explain this phenomenon, one can only speculate about (other) factors which may have determined the different outcome in the ex vivo pemphigoid model. Nevertheless, the low or absent capacity of autoantibodies against the epidermal basement membrane to induce dermal-epidermal separation ex vivo parallels the lack of blisters at the time point of diagnosis in patients.

The BP treatment modalities were different in all patients, based on the guidelines for treatment of autoimmune blistering disorders. None of the patients responded sufficiently to topical treatment with clobetasolpropionate ointment alone. Low-dose oral prednisolone in combination with a steroid-sparing agent was necessary. This improved the ARPD-like skin lesions in all cases.

Perforating diseases comprise a group of pruritic skin disorders characterized by extruding connective tissue through the epidermis. Two main types have been described in which collagen and/ or elastic fibers perforate the epidermis ${ }^{8}$. ARPD occurring during adulthood is 
most commonly associated with diabetic nephropathy, renal failure, hypertension and chronic venous insufficiency ${ }^{5}$. Several constituents of the extracellular matrix, among them laminin332, collagen type IV, tenascin and fibronectin were found in a soluble form within serum of patients affected by ARPD ${ }^{9}$. Although not previously shown, BP180 and BP230 might also be involved in this process, thus being exposed to the immune system and inducing an autoimmune response. BP can also manifest as highly pruritic prurigo nodularis (PN)-like eruptions with clinical and histological features consistent with PN. PN lesions can precede the onset of blistering, in other cases the lesions occur in parallel. In has been suggested that ARPD is a variant of $P N^{10}$.

Our results highlight that ARPD is a clinical manifestation, characterized by the RPC histopathological pattern, which can represent a nonbullous BP variant with significant differences to classical BP, suggesting a distinct pathogenetic background. Thus, in ARPD patients BP diagnostics should be initiated.

\section{ACKNOWLEDGEMENTS}

We thank the patients. We thank Annegret Bedorf for the excellent technical assistance. DK's work has been supported by the German Research Foundation DFG (KI1795/1-1), EB research Partnership and the Mathilde-Wagner-Habilitationspreis from the University of Freiburg.

\section{Figure legends:}

Figure 1. (a) Direct immunofluorescence with $\lg$ and $C 3 c$ in cryosections of patient 1 shows linear deposition at DEJZ. (b) Clinical presentation of patients 1-5 and data on total $\lg$ E levels and $\lg$ / $\lg$ E autoantibodies against BP180 / BP230 at time of BP diagnosis. Patients 1-4 presented with generalised erythematous papules and nodules with central necrotic plugs, only patient 5 had widespread urticarial plaques and large tense blisters at initial presentation. Clinical features of ARPD were visible as a healing pattern at her chest, back and legs. (c) Histological and immunohistological presentation of ARPD in formalinfixed, paraffin-embedded patient tissue of patient 1. Haematoxylin- eosin (HE) staining reveals invagination of the epidermis filled with a small plug consisting of keratin and cellular debris, transepidermal elimination of collagen bundles (100x) and a perivascular neutrophilic infiltrate with sparce eosinophils (400x). Elastica van Gieson (EvG) staining highlights vertically orientated bundles of collagen fibers beneath the necrotic plug (100x). The graph displays a quantification of the neutrophils versus the eosinophils in the inflammatory 
infiltrate of the histology from patients 1 and 2. For the analysis 10 fields were investigated in total from both biopsies, the difference is statistically significant $\left.{ }^{\star * *} \mathrm{P}<0.0001\right)$.

Supplemental Figure 1. (a) The indirect immunofluorescence showed IgG depositions on the blister roof in all patients. As a positive control the serum of a patient with highly-active BP has been used. (b) BP autoantibodies induce subepidermal splits. Cryosections of normal human skin were incubated with human serum samples and, in a second step, with leukocytes from healthy donors. In contrast to serum from a healthy control subject, incubation with serum from a patient with full-blown BP resulted in extensive dermalepidermal separation. Serum samples from patients evaluated in the present study showed different pathogenic potential in this assay, including no subepidermal splits (patient 2), as well as slightly different degrees of modest dermal-epidermal separation in the other patients (patients 1,4,5).

\section{References}

1 Bakker CV, Terra JB, Pas HH, Jonkman MF. Bullous pemphigoid as pruritus in the elderly: a common presentation. JAMA Dermatol 2013; 149:950-3.

2 Lamberts A, Meijer JM, Jonkman MF. Nonbullous pemphigoid: A systematic review. J Am Acad Dermatol 2018; 78:989-995.e2.

3 Nomura H, Mukai M, Niimi Y, et al. Coexistence of acquired perforating dermatosis and bullous pemphigoid: three cases. Eur J Dermatol EJD 2017; 27:192-3.

4 Tani S, Ishii N, Hashimoto T, Tsujioka K. Bullous pemphigoid arising in a patient with acquired perforating dermatosis. Clin Exp Dermatol 2017. doi:10.1111/ced.13080.

5 García-Malinis AJ, Del Valle Sánchez E, Sánchez-Salas MP, et al. Acquired perforating dermatosis: clinicopathological study of 31 cases, emphasizing pathogenesis and treatment. J Eur Acad Dermatol Venereol JEADV 2017. doi:10.1111/jdv.14220.

6 Izumi K, Nishie W, Mai Y, et al. Autoantibody Profile Differentiates between Inflammatory and Noninflammatory Bullous Pemphigoid. J Invest Dermatol 2016; 136:2201-10. 
7 Sitaru C, Schmidt E, Petermann S, et al. Autoantibodies to bullous pemphigoid antigen 180 induce dermal-epidermal separation in cryosections of human skin. J Invest Dermatol 2002; 118:664-71.

8 Bolognia JL. Dermatology., Elsevier; 4 edition.

9 Morgan MB, Truitt CA, Taira J, et al. Fibronectin and the extracellular matrix in the perforating disorders of the skin. Am J Dermatopathol 1998; 20:147-54.

10Kestner R-I, Ständer S, Osada N, et al. Acquired Reactive Perforating Dermatosis is a Variant of Prurigo Nodularis. Acta Derm Venereol 2017; 97:249-54.

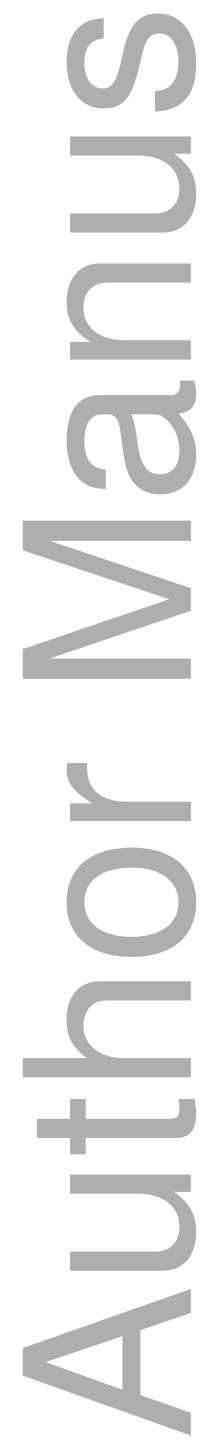




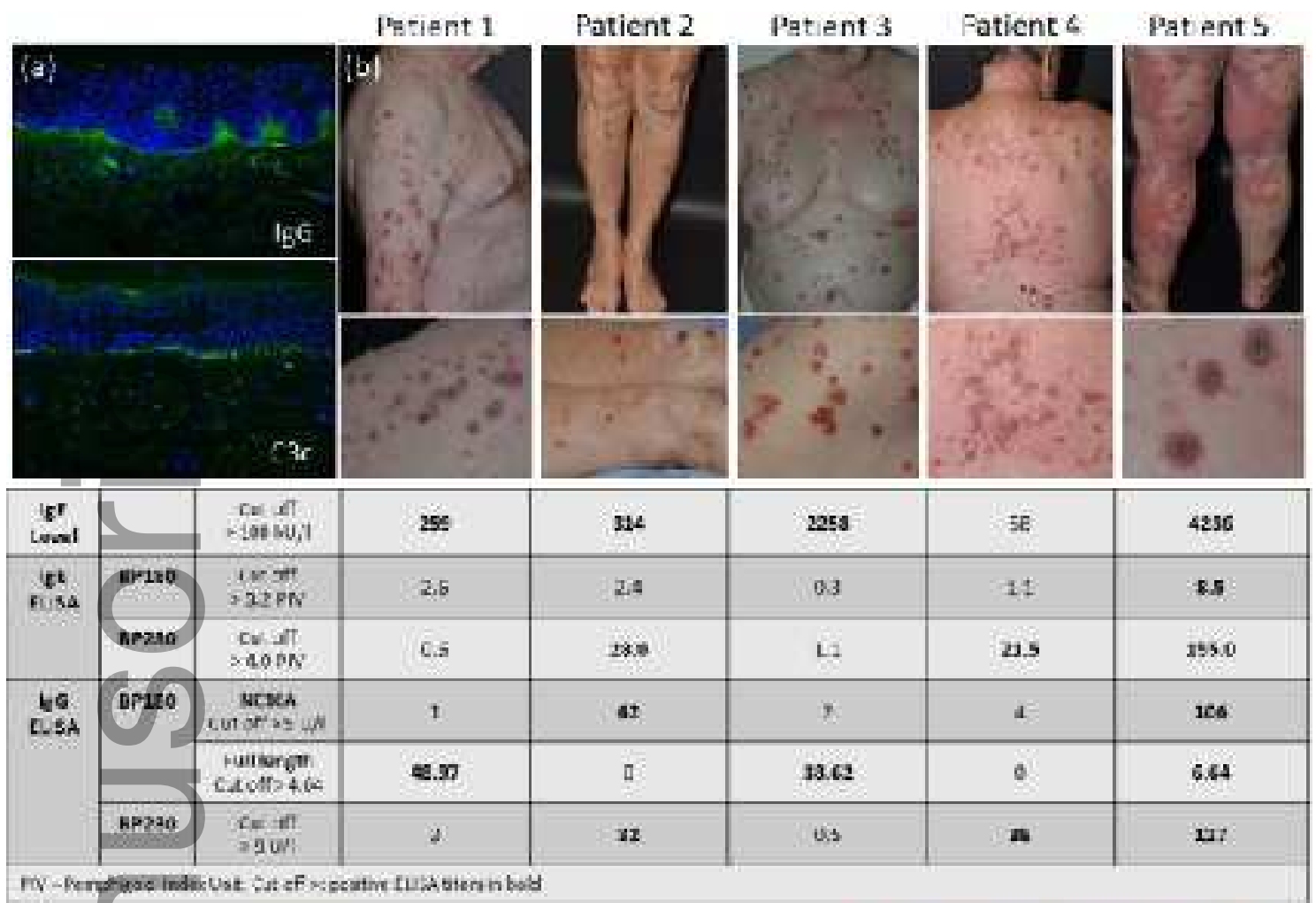
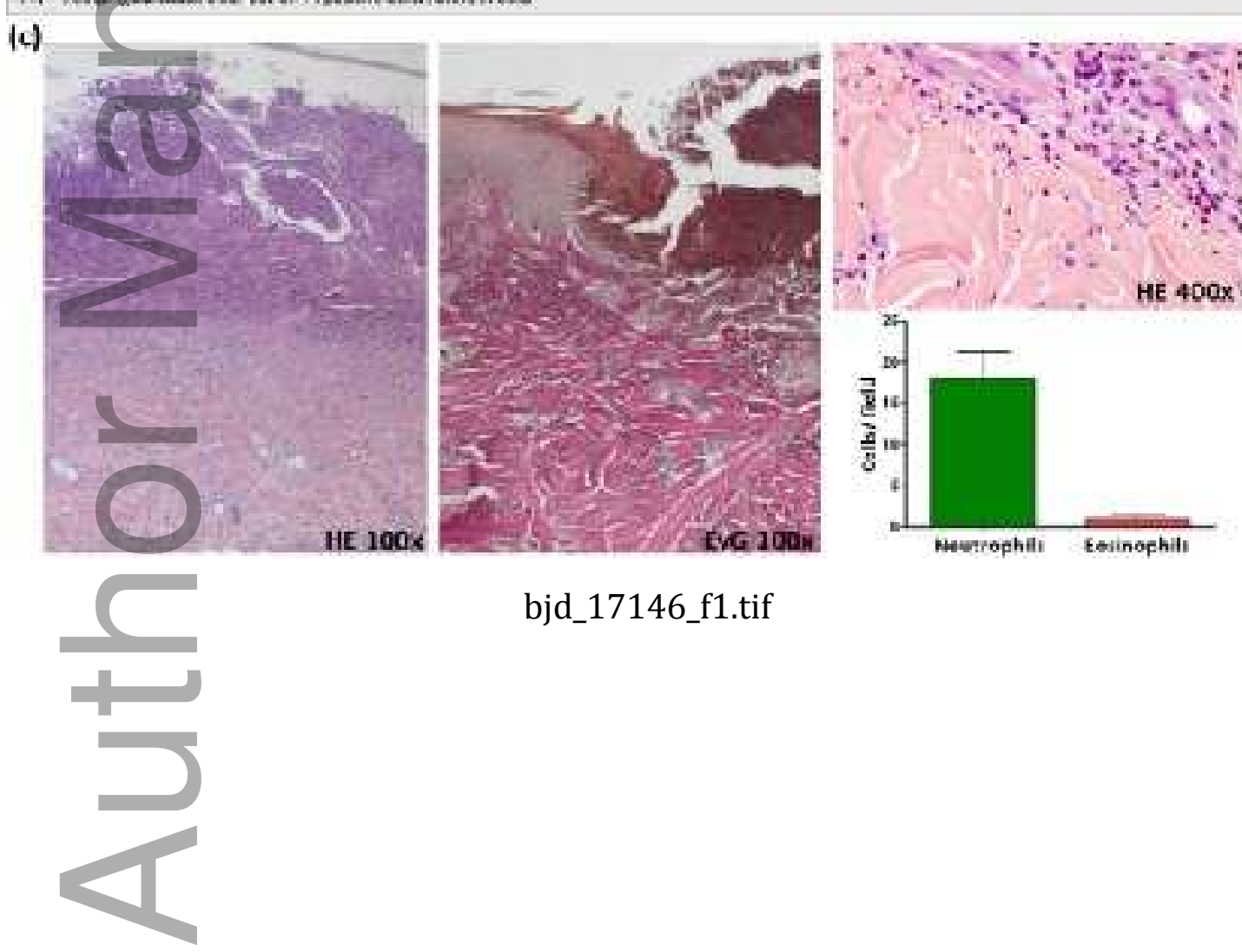

bjd_17146_f1.tif 


\section{University Library}

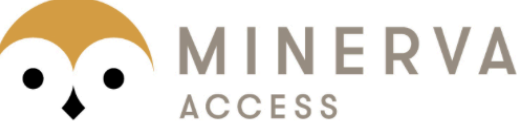

A gateway to Melbourne's research publications

Minerva Access is the Institutional Repository of The University of Melbourne

Author/s:

Schauer, F;Kern, JS;Virtic, O;Technau-Hafsi, K;Meiss, F;Thoma, K;Athanasiou, I;Sitaru, C;Di Zenzo, G;Izumi, K;Nishie, W;Shimizu, H;Bruckner-Tuderman, L;Kiritsi, D

Title:

A new clinical variant of acquired reactive perforating dermatosis-like bullous pemphigoid

Date:

2019-01-01

\section{Citation:}

Schauer, F., Kern, J. S., Virtic, O., Technau-Hafsi, K., Meiss, F., Thoma, K., Athanasiou, I., Sitaru, C., Di Zenzo, G., Izumi, K., Nishie, W., Shimizu, H., Bruckner-Tuderman, L. \& Kiritsi, D. (2019). A new clinical variant of acquired reactive perforating dermatosis-like bullous pemphigoid. BRITISH JOURNAL OF DERMATOLOGY, 180 (1), pp.231-232. https:// doi.org/10.1111/bjd.17146.

Persistent Link:

http://hdl.handle.net/11343/285130 